\title{
Gesteuertes Selbststudium mit Lernportfolios
}

\author{
Verena Rengglia,c, Regina Kunz ${ }^{a, c}$, Elisabeth Zemp ${ }^{b, c}$, Yvonne Bollaga,c \\ ${ }^{a}$ asim Versicherungsmedizin, Universitätsspital Basel; ${ }^{b}$ Schweizerisches Tropen- und Public Health-Institut; ${ }^{\circ}$ Universität Basel
}

\begin{abstract}
Von Studierenden und Mentoringpersonen wurde es als Bereicherung empfunden: das Lernportfolio, eine an selbstgewählten Lernzielen orientierte, strukturierte Sammlung von studentischen Aktivitäten mit geplanter Selbstreflexion. Es gilt als modernes Instrument zur Steuerung des Selbststudiums und der professionellen Entwicklung.
\end{abstract}

\section{Hintergrund}

Die postgraduale Weiterbildung ermöglicht die Vertiefung von Themen, die während der Ausbildung nur ansatzweise gelehrt werden und die Aneignung von Wissen und Kompetenzen, die praktische Arbeitserfahrung voraussetzen. Sie ist ein wichtiger Bestandteil des lebenslangen Lernens. Je heterogener die Zielgruppe, an die sich ein postgraduales Weiterbildungsprogramm wendet, umso schwieriger ist es jedoch, allen spezifischen Bildungsbedürfnissen der Teilnehmenden gerecht zu werden. Hier kann die Methode des gesteuerten Selbststudiums mit Lernportfolio sinnvoll zum Einsatz kommen $[1,2]$. Die Methode eignet sich auch, die Vernetzung von Wis-

\section{Travail personnel guidé grâce au portefeuille d'apprentissage}

La méthode du travail personnel guidé grâce à un portefeuille d'apprentissage vise à encourager les échanges de connaissances et la mise en pratique des nouveaux acquis dans le quotidien professionnel, en d'autres termes le transfert entre théorie et pratique. Le présent article revient sur les expériences réalisées avec cette méthode dans le cadre du master en médecine des assurances, une formation postgrade offerte par l'Université de Bâle. Pour cela, les étudiants ont été interrogés sur leurs attentes à l'égard du portefeuille, leurs expériences, appréhensions et utilisation personnelle, à l'aide d'un bref questionnaire structuré. Au terme de ce travail personnel guidé, trois quarts des étudiants considèrent le portefeuille comme une plus-value pour leur formation, un avis partagé par leurs mentors qui recommandent cette méthode afin d'encourager un processus de formation actif, autonome et suscitant la réflexion. sen zu fördern und die Integration von neu Erlerntem in den Arbeitsalltag herzustellen und somit den Theorie-Praxis-Transfer zu unterstützen [1]. Dieser Artikel beschreibt und diskutiert die Erfahrungen mit der Lernportfolio-Methode, die im Rahmen des Masters in Versicherungsmedizin (MAS VMed) gemacht wurden, der an der Universität Basel als berufsbegleitender, modular aufgebauter Studiengang angeboten wird.

Der Masterstudiengang integriert versicherungsmedizinische Kerninhalte und Kompetenzen [3]: Krankheitsbilder aus versicherungsmedizinischer Perspektive, juristische Grundlagen und Versicherungsrecht, handlungsbezogenes medizinisches Wissen, Krankschreibung und Förderung der Rückkehr zur Arbeit, Begutachtung, Steuerung im Gesundheitssystem und in der Krankenversorgung, Risikoeinschätzung für Lebensversicherung, Förderung von Teilhabe in der Gesellschaft jenseits des Arbeitslebens. Gelehrt wird mit Dozierenden aus verschiedenen Disziplinen wie Medizin, Versicherungsund Gesundheitsrecht und -ökonomie, Ethik, Public Health, Epidemiologie, Arbeitsmedizin und Versicherungsmathematik. Das Programm wendet sich schwerpunktmässig an Mediziner sowie an Fachpersonen weiterer für die Versicherungsmedizin relevanter Fachrichtungen wie Juristen, Ökonomen und Fachexperten der Versicherungswirtschaft. Um nebst den curricularen Inhalten auch die individuellen Lerninteressen der Kursteilnehmenden abbilden zu können, wurde das Lernportfolio in die Struktur des Studiengangs eingebaut. 


\section{Das Lernportfolio als methodisches Werkzeug}

Das Lernportfolio, eine an selbstgewählten Lernzielen orientierte, strukturierte Sammlung von studentischen Aktivitäten mit geplanter Selbstreflexion [4], gilt als modernes Instrument zur Steuerung des Selbststudiums und der professionellen Entwicklung $[1,5]$.

Das Lernportfolio im MAS VMed hat folgende Struktur: Zu Beginn des Studiengangs identifizieren die Teilnehmenden zwei bis drei Fachthemen, mit denen sie sich in den kommenden Jahren vertieft auseinan-

\section{Die fortlaufende Dokumentation der Lern-} aktivitäten dient auch einer kritischen Auseinandersetzung mit dem neu Gelernten.

dersetzen möchten. Sie entwickeln spezifische Lernziele, die als Leitplanken für den weiteren Lernprozess dienen. Beispiele dazu finden sich in Übersicht 1. Anhand der formulierten Lernziele stellen sich die Teilnehmenden ihre eigenen Lernaktivitäten zusammen, führen diese durch und dokumentieren sie. Dies kann beispielsweise die Lektüre von Fachartikeln sein, die Teilnahme an Kursen oder Fortbildungsveranstaltungen oder das Führen von Expertengesprächen. Die fortlaufende Dokumentation der Lernaktivitäten dient auch einer kritischen Auseinandersetzung mit dem neu Gelernten.

Übersicht 1: Beispiele von Lernzielen, die von Teilnehmenden des MAS VMed gewählt wurden

- Leistungspflicht der verschiedenen (Sozial)-Versicherungen bei Spitalleistungen kennen und im Leistungsfall anwenden können.

- Managed Care / Case Management in Deutschland, Österreich und Schweiz beurteilen können.

- Psychiatrische Gutachten auf formale und inhaltliche Qualität sicher beurteilen können.

Stellungnahmen in komplexen Arzthaftpflichtfällen kompetent ausführen können.

- Verschiedene Präventionsprogramme bewerten können.

Um die Verbindung der individuellen Lernziele des Lernportfolios mit dem Curriculum des Masterstudiums herzustellen, verfassen die Teilnehmenden nach jeder der 14 Präsenzwochen eine kurze Reflexion, wie die Inhalte der Kurswoche zur Erreichung der Lernziele ihres Portfolios beigetragen haben.

Jeder Teilnehmende erhält eine Mentoringperson zugeteilt, die das Portfolio-Lernen während der gesamten Dauer begleitet und eine prozessorientierte Unterstützung bietet. Einmal pro Semester findet ein persönliches Gespräch statt. Dabei wird der Lernpro- zess gemeinsam reflektiert, das Erreichte mit den Lernzielen abgeglichen, und das weitere Vorgehen besprochen.

\section{Erste Erfahrungen}

Im April 2011 startete der erste Durchgang des MAS VMed mit 15 Teilnehmenden. Zu Beginn des Studiengangs und nach Abschluss des Lernportfolios erfolgten jeweils kurze, strukturierte schriftliche Befragungen unter den Studierenden über ihre Erwartungen an das Portfolio, ihre Erfahrungen, Befürchtungen und ihr persönliches Vorgehen.

Bei Studienstart verfügte keiner der Teilnehmenden über Erfahrungen mit der Erstellung eines Lernportfolios. Als Erwartungen formulierten die Teilnehmenden hauptsächlich die mögliche Vertiefung der eigenen Lerninteressen, das Setzen eigener Schwerpunkte und das selbständige Arbeiten (Übersicht 2). Bedenken betrafen vor allem eine mögliche Verzettelung bei der Bearbeitung der Lernziele, eine zu breite Themenwahl und einen zu grossen Zeitaufwand.

Übersicht 2: Erwartungen der Teilnehmenden

an das Lernportfolio bei Studienbeginn

- Im Sinne eines roten Fadens, die selbstfestgelegten Hauptthemen zielgerichtet bearbeiten und notwendige Fähigkeiten erwerben zu können.

- Ich finde es positiv, die eigenen Schwerpunkte setzen zu können, v.a. da die Gruppe sehr heterogen ist. Ich finde es spannend, meine Lernplanung mehr oder weniger selbständig zu gestalten. Viele Inputs werden durch die Module abgedeckt. Das Portfolio lässt der Kreativität freien Lauf.

- Durch das Lernportfolio kann ich meine selbstgewählten Lernziele besser erreichen, da ich mich vermehrt damit auseinandersetzen muss, insbesondere auch durch die Reflexion nach jedem Modul.

- Freie Zeiteinteilung.

Nach erfolgreichem Abschliessen des Portfolios beurteilten drei Viertel der Absolvierenden das Lernportfolio als Mehrwert für ihr persönliches Lernen im Studiengang. Eine Auswahl der von ihnen genannten positiven Aspekte findet sich in Übersicht 3.

Als Limitationen empfanden die Teilnehmenden den hohen Zeitaufwand, die lange Zeitspanne, bis der eigene Arbeitsstil gefunden wurde, und das Fehlen von Beispielen für eine mögliche Ausgestaltung eines Lernportfolios. Die zu Beginn des Studiums verteilte Wegleitung zum Lernportfolio wurde in einigen Punkten als unklar empfunden.

An der Schlussbesprechung beurteilten auch die begleitenden Mentoringpersonen das Lernportfolio als Bereicherung für den Studiengang. Je nach Themen- 
Übersicht 3: Von den Teilnehmenden formulierte positive Aspekte des Lernportfolios

- Ich konnte Themen bearbeiten, welche für mich wirklich von Nutzen sind.

- Interessante Gespräche wurden möglich; viele Türen konnten geöffnet werden.

- Die Motivation, in der Entwicklung und Umsetzung des Case Management involvierte Personen persönlich zu befragen und hierdurch unerwartete Erkenntnisse zu erhalten.

- Das «Sich-Zeit-nehmen-Müssen` für eine Thematik, die interessiert, wo ich ohne Verpflichtung zur Bearbeitung aber wahrscheinlich die Initiative zur Abklärung des Themas in dieser Form nicht in Angriff genommen hätte.

- Beim gezielten Lernen (Lesen, Zuhören, Nachfragen, Se minarbesuche, Beobachten, Vertiefen usw.) habe ich in meinen Themen die Zusammenhänge besser kennengelernt.

wahl der Studierenden wurden sie mit neuen Themen konfrontiert, was auch bei ihnen einen Lernprozess auslöste. Die Semestergespräche mit den Teilnehmenden waren meist anregend. Am schwierigsten wurde die Startphase beurteilt, bis die Lernziele von den Teilnehmenden richtig und - vor allem bezüglich des Umfangs - realistisch ausformuliert waren.

\section{Diskussion}

Die Erstellung eines Lernportfolios im Rahmen eines postgradualen Masterstudiengangs wird von der Mehrheit der Studierenden als Mehrwert eingeschätzt, da damit individuelle Lernziele und Interessen abgedeckt werden können. Auch der TheoriePraxis-Transfer wird unterstützt. Zudem offeriert die Methode die Möglichkeit, Lernaktivitäten, die den Teilnehmenden in ihrem Arbeitsalltag auferlegt werden, strukturiert und effizient in die Weiterbildung zu integrieren.

Neben der Erarbeitung der Inhalte spielt beim Lernportfolio die Reflexion des eigenen Lernprozesses eine wichtige Rolle. Unter Reflexion versteht man dabei den mentalen Prozess, bei dem eine Erfahrung, ein Problem oder bestehendes Wissen (neu) strukturiert wird. Dadurch kann die eigene Entwicklung besser verstanden und der Lernprozess besser geplant werden [6]. Ist die Methode der Reflexion nicht vertraut, kann sie jedoch zu Irritationen führen, besonders wenn Reflexionen schriftlich verfasst werden sollen [4]. In unserem Studiengang haben wir bei Auftreten solcher Irritationen oder Verunsicherungen über die zu verfassenden Reflexionen versucht, in persönlichen Gesprächen mit den Teilnehmenden und mit der Abgabe von Beispielen motivierende Unterstützung zu leisten.
Die Abgabe einer Wegleitung mit klaren Informationen bietet den Studierenden eine wichtige Orientierungshilfe $[1,5]$. Nach Rückmeldungen der Teilnehmenden konnten wir unsere Wegleitung für das Lernportfolio gezielt überarbeiten und unklare Punkte konkretisieren. Auch hatten wir ab dem zweiten Studiengang die Möglichkeit, ein komplettes Portfolio als Beispiel zur Verfügung zu stellen. Im Weiteren optimierten wir die Einführungsveranstaltung zum Lernportfolio, in welchen ein Workshop zum Thema «Formulieren von Lernzielen» eingebaut wurde.

Von grosser Wichtigkeit in der erfolgreichen Umsetzung der Methode des Lernportfolios ist die individuelle Betreuung der Studierenden durch persönlich zugeteilte Mentoringpersonen [4, 6]. Diese verstehen sich als "Prozessbegleitende», sie unterstützen die Studierenden beim Erarbeiten der Lernziele und geben Input, der die Studierenden anregen soll, andere Blickwinkel wahrzunehmen.

Lernportfolios können auch in der ärztlichen Fortbildung eingesetzt werden [7]. Auch hier stützt sich das Konzept darauf, dass eigene Wissensdefizite identifiziert werden und darauf aufbauend ein persönlicher Lern-/Fortbildungsplan erstellt wird. Damit fördert das Lernportfolio als methodisches Werkzeug einen aktiven, selbstbestimmten, und reflektierenden Bildungsprozess.

\section{Hinweis}

Master of Advanced Studies Versicherungsmedizin (MAS VMed). Der postgraduale Studiengang wird berufsbegleitend über 3 Jahre an der Universität Basel angeboten. Der nächste Durchgang startet am 31. August 2015. Weitere Informationen sind erhältlich unter www.usb.ch/asim oder www.swisstph.ch/de/ausbildung/ professional-postgraduate.htm

\section{Referenzen}

1 Landwehr N, Müller E. Das Portfolio - ein Instrument zur individualisierten Steuerung und Beurteilung des (Selbst-) Studiums. In: Begleitetes Selbststudium. Bern: hep Verlag ag; 2008:74-85

2 Zubizarreta J. The Learning Portfolio: a powerful idea for significant learning. Idea Paper \#44 (Internet). 2008. (zitiert am 17.12.2013. www.theideacenter.org/sites/default/files/IDEA_ Paper 44.pdf

3 Renggli V, Bollag Y, Schmidts C, Lehr M, Brugger C, Kunz R. Versicherungsmedizinische Kompetenz per Mausklick für den ärztlichen Alltag. Schweiz Ärztezeitung. 2013; 94(43):1613-5.

4 Schmidt A, Hahn EG. Entwicklung und Implementierung eines Portfolio-basierten Ausbildungsprogramms für das Tertial Innere Medizin des praktischen Jahres. GMS Z Med Ausbild. 2009;26(1):Doco9.

5 Driessen EW, van Tartwijk J, Vermunt JD, van der Vleuten CPM. Use of portfolios in early undergraduate medical training. Med Teach. 2003;25(1):18-23.

6 Driessen E, van Tartwijk J, van der Vleuten C, Wass V. Portfolios in medical education: why do they meet with mixed success? A systematic review. Med Educ. 2007;41:1224-33.

7 Donner-Banzhoff N, von Luckner A, Wilm S. Moderne Formen der Fortbildung. Dtsch Arztbl. 2005;102:A818-9. 